\title{
Data Uncertainty Assessment and Information Flow Analysis for Product-Service Systems in a Library Case Study
}

\author{
Durugbo Christopher*, Erkoyuncu John A., Tiwari \\ Ashutosh, Alcock Jeffrey R., Roy Rajkumar, Shehab \\ Essam \\ School of Applied Sciences, \\ Cranfield University, \\ Bedfordshire, \\ MK43 0AL, United Kingdom \\ E-mail: \{c.durugbo, j.a.erkoyuncu, a.tiwari, j.r.alcock, r.roy, e.shehab \\ @ cranfield.ac.uk \\ ${ }^{*}$ Corresponding author
}

\begin{abstract}
The provision of service is increasingly becoming a key driver for delivering customer value. This emphasis on service provision is promoted by approaches such as Product-Service Systems (PSSs) that delivers value in use based on integrated products and services. However, to achieve effective delivery of services, it is important to manage information flow and data uncertainty associated with information requirement. The paper proposes a methodology that consists of: (a) the assessment of data uncertainty through the numeral, unit, spread, assessment and pedigree approach and a pedigree matrix consisting of data uncertainties to be scored based on their influence on the system; and (b) the analysis of information flow by means of data flow diagrams that depict information exchanges and an Info-dynamic engine that measures the efficiency and recommends improvements for information flows in PSSs. A library case study was undertaken to show how such a methodology can be applied.
\end{abstract}

Keywords: Data Uncertainty; Product-Service Systems; Information Flow; Service; Library system

Biographical notes: Christopher Durugbo is a $\mathrm{PhD}$ researcher at the School of Applied Sciences at Cranfield University. His research interests include microsystems production, engineering design, information and technology management, and human factors.

John Ahmet Erkoyuncu is a PhD researcher at the School of Applied Sciences at Cranfield University. His research interests include Product-Service Systems, cost estimation, modelling and managing uncertainty.

Ashutosh Tiwari is a Reader in Engineering Optimisation and Head of the Decision Engineering Centre at Cranfield University. Dr Tiwari's interests include product/process design optimisation, evolutionary computing and product-service system design.

Jeffrey R Alcock is a Reader in Materials at Cranfield University. Dr Alcock 
has academic, research and industrial background in product-service systems and micro-device design.

Rajkumar Roy led Competitive Design research at Cranfield for over ten years before his promotion to the Head of Manufacturing Department in 2009. Professor Rajkumar Roy's research interests include qualitative cost modelling, requirements management and design optimisation research.

Essam Shehab is a Senior Lecturer in Decision Engineering and is leading cost engineering research at Cranfield University. Furthermore Dr. Shehab has an academic, research and industrial background in product-service systems and knowledge-based decision support.

\section{Introduction}

A Product-Service System (PSS) is a customer-focused approach proposed as "an integrated product and service offering that delivers value in use" (Baines et al., 2007). Product-Service Systems are 'social constructs' (Morelli, 2006) for realising goals, delivering results and solving problems. To do this, information needs to be gathered during various stages of a PSS. Much like service, information is non-physical, requires an environment, a channel and content. Providing information in itself is usually a service but service artefacts may not offer information. Information can be described as useable data, inferences from data, or data descriptions (Ackoff, 1989). However, unlike services, information flow and exchanges especially in organisations usually require some form of integrity, confidentiality and security.

Analysing information flow in a PSS is vital in understanding interactions involving customers and providers (Durugbo et al., 2010a). These interactions are essential in the design and delivery of a PSS for three main reasons: to design and enable communications and networks for interdisciplinary access/exchange of information and for life cycle management, to outline information systems for enabling PSS delivery, and to propose information flow models that aid service designers in the conceptual design of a PSS. A PSS, therefore is not only made up of a product and a service, it also needs to consider information requirements to aid the delivery process in an affordable (for the customer) and profitable (for the provider) manner.

This study is motivated by the need to consider information requirements for a PSS in terms of information flow and data uncertainty. Ormerod and Shepherd (2004) defined information requirement as consisting of data required to realise a goal safely and efficiently. Information requirement also defines the content of an information system and is determined based on system analysis and design activities.

The aim of the paper is to identify and apply an approach for assessing data uncertainty and analysing information flow in a PSS. The paper will consider this by investigating the factors influencing data uncertainty and how this affects information flows for a PSS.

The remainder of this paper has been structured as follows. Section 2 describes the methodology applied for the research while Section 3 makes use of literature as a background to outline an approach for assessing data uncertainty and for analysing information flow in systems. Section 4 presents a case study in which the proposed approach is demonstrated. Section 5 presents the findings of the case study while the 
benefits of the approach, implications for PSS design and limitations of the research are outlined in Section 6.

\section{Methodology}

The research was conducted in two phases: literature background and case study.

Literature background: During this phase, literature was reviewed to identify an approach for assessing data uncertainty and analysing information flow in a PSS. The numeral, unit, spread, assessment and pedigree (NUSAP) approach developed by Funtowicz and Ravetz (1990) was adopted for assessing data uncertainty. This approach was selected due to its benefit in systematically capturing the influence of uncertainty by eliciting expert opinion and representing it quantitatively. The Info-dynamic engine proposed by Sundresh (1997) was selected as the scheme for analysing information flow based on three factors: efficiency, adaptability and reusability.

Case study: For this phase of the research, pre-defined sets of questions were used to conduct semi-structured interviews with PSS providers about a PSS function involving data and information flow. In the case study, the PSS provider was the Cranfield University Library (CUL) whereas the PSS function was an interlibrary loan request service. A total of four semi-structured interviews were conducted with all three members of the CUL-interlibrary loan department. Each interview session lasted for an hour and the research also benefitted from documents that were provided by the participants. The content varied, including: list of uncertainties and number of interlibrary loan requests over 2 months.

\section{Literature Review}

\subsection{Information Needs for Product Service Systems}

Within the context of a PSS, information about the domain of application, nature of business and available resources is required for determining service strategies (Durugbo et al., 2009). Information for use in PSS design and delivery could include customer records, machine records, service team records, business records and sales records.

The flow of information in a PSS is major factor that contributes to: minimising resource inputs and maximising outputs in the form of value (Baines et al., 2007) and enabling communication by means of face-to-face interaction and media forms (Durugbo et al., 2010a). The manner in which information flows in a PSS also determines key management strategies for life-cycles, business processes, value nets, supply chains and so on (Mont, 2002, Roy and Cheruvu, 2010).

The analysis of information flow in a PSS is therefore a major design challenge that requires effective techniques for modelling information exchanges (Durugbo et al., 2010b). The challenge is also borne from the fact that any strategy adopted for managing production and manufacturing depends on how a product is positioned within its life cycle (Meade and Rabelo, 2004). This is because product markets are usually complex and fundamentally non-linear in nature. This makes the task of forecasting market trends or direction a difficult activity to carry out objectively. Furthermore, service designers are required to be innovative in the design of services throughout the life-cycle of a product to maintain competitiveness in the market (Verganti and Buganza, 2006). 


\subsection{Data Uncertainty Assessment}

Data uncertainty refers to things that are not known or known imprecisely due to issues existing in data. This largely influences the quality of data, which has an influence on the effective use of information systems (Coulon et al., 1997). This in essence affects organisation's performance as the ability to make decisions, and communicate unique, non-routine, consequential events diminishes. Uncertainty is knowledge driven and has typically been associated with the inability to predict an outcome (Erkoyuncu et al., 2009a). For instance in the case of cost, data may relate to the cost breakdown structure (e.g. research and development, production and construction, operations and maintenance, retirement and disposal costs) of a given project (Curran et al., 2004). The level of manageable uncertainty can particularly be driven by the process of transforming data into information and capturing knowledge from available information (Erkoyuncu et al, 2010). Uncertainty creates challenges in determining the sufficient level of information requirements for a certain activity.

Highly sensitive variables create variability in data where some examples include frequency of the maintenance activity, variation of the assets utilisation or operating time, variation of corrective maintenance hours per operating hour, product demand rate, product distribution time and the discount rate (Emblesvag, 2003). However, the uncertainty issue is predominantly driven by the data collection and storage processes.

Examples of data uncertainty categories include reliability (i.e. precision, credibility, uncertainty of the information), completeness (i.e. data gaps, inconsistent data definitions), accessibility (i.e. available, data communication, access issues, wrong format), relevance (i.e. useful), representative (i.e. applicability due to differences between projects or organisations in time, technology and geographical coverage...), repeatability (i.e. variation in learning curves, consistency and reproducibility of processes in data collection) (Flanagan et al., 1989; Boussabaine and Kirkham, 2004).

For data uncertainty there is little consensus in how to define the relevant sources of uncertainties, their characteristics and how the characteristics can be related for uncertainty to be treated or managed. Whilst the uncertainties vary depending on the specific context, a list of commonly utilised methods and tools used to assess uncertainties are explained in Refsgaard et al. (2007). This paper adopts the NUSAP approach, which refers to 5 qualifiers that are used to qualify quantities: numeral, unit, spread, assessment and pedigree. The approach is capable of combining qualitative analysis (numeral, unit, spread) and the systematic multi-criteria evaluation of a given knowledge base (pedigree) (Van der Sluijs et al., 2003). It offers both qualitative and quantitative dimensions of uncertainties to be displayed in a standardised and selfexplanatory manner (Van Krauss et al., 2008).

Pedigree contains a set of criteria that is used to assess a number of aspects related to the information flow and knowledge used. This approach aims to define the strength of the parameters that are assessed. Assessment of the pedigree criteria necessitates subjective input, which makes it hard to capture objective input. However, Funtowics and Ravetz (1990) highlight the high degree of agreement on the pedigree ratings among experts within the general area of competence. This is driven by the pedigree matrix that reduces the subjectivity by defining each criterion in discrete figure from 0 (high uncertainty) to 4 (low uncertainty) with linguistic descriptions for each level of scale (Van der Sluijs et al., 2005). 
As a result, the pedigree is considered to be a robust indicator of the strength of the associated NUSAP assessment. The pedigree criteria covered in this paper are empirical basis, methodological rigour, and validation, as represented in Table 1 . This set of criteria was selected bearing in mind the requirements in terms of data uncertainty in a PSS. Empirical basis assesses the degree of direct observations, measurements and statistics that are used in estimating a parameter. Methodological rigour refers to the quality in measuring and processing the data. Validation considers the level of ability to crosscheck the data and assumptions used to produce the figure of the parameter against independent sources. This approach requires the identification and mapping of uncertainties. This is commonly achieved through an expert elicitation workshop (Stewart et al., 1995). The objective of the uncertainty assessment is to establish an understanding of the uncertainties that are high in importance and impact.

Table 1 Uncertainty assessment criteria: Pedigree Scoring

\begin{tabular}{|c|c|c|c|}
\hline Score & Empirical & Methodological rigour & Validation \\
\hline 4 & $\begin{array}{l}\text { Large sample direct } \\
\text { measurement of } \\
\text { controlled experiments } \\
\text { are carried out }\end{array}$ & $\begin{array}{l}\text { Best available practice in well } \\
\text { established discipline }\end{array}$ & $\begin{array}{l}\text { Compared with independent } \\
\text { measurements of the same } \\
\text { variable over long domain }\end{array}$ \\
\hline 3 & $\begin{array}{l}\text { Historical field data and } \\
\text { small sample direct } \\
\text { measurements }\end{array}$ & $\begin{array}{l}\text { Reliable method common } \\
\text { within established discipline, } \\
\text { best available practice in } \\
\text { immature discipline }\end{array}$ & $\begin{array}{l}\text { Compared with independent } \\
\text { measurements of closely } \\
\text { related variable over shorter } \\
\text { period }\end{array}$ \\
\hline 2 & $\begin{array}{l}\text { Modelled/derived data; } \\
\text { indirect measurement }\end{array}$ & $\begin{array}{l}\text { Acceptable method but } \\
\text { limited consensus on } \\
\text { reliability }\end{array}$ & $\begin{array}{l}\text { Measurements no } \\
\text { independent, proxy variable, } \\
\text { limited domain }\end{array}$ \\
\hline 1 & $\begin{array}{l}\text { Educated guesses, } \\
\text { indirect approximate } \\
\text { rule of thumb estimate }\end{array}$ & $\begin{array}{l}\text { Preliminary methods, } \\
\text { unknown reliability }\end{array}$ & $\begin{array}{l}\text { Weak very indirect } \\
\text { validation }\end{array}$ \\
\hline 0 & Crude speculation & No discernible rigour & No validation performed \\
\hline
\end{tabular}

\subsection{Information Flow Analysis}

Information flow is based on information exchanges in a system and is associated with data flow and system processes (Durugbo et al., 2010b). The purpose of information exchanges in a system is to communicate knowledge and understanding (Scheller, 1990). The idea is that data descriptions (information) must be uniform in the way the receiver interprets the data and the manner in which the sender meant the information to be interpreted. Information exchanges in a PSS can be represented by a Data Flow Diagram (Durugbo et al., 2009a). Data flow diagrams were developed by DeMarco, (1979) in the late seventies and are very popular tools applied in industry for the development of systems. They are made up of four main components (Durugbo et al., 2010b):

- $\quad$ Rounded rectangles that represent processes (or functions);

- Rectangles that depict external entities;

- $\quad$ Open ended rectangles that indicate data stores; and 
- Arrows that stand for data or information flow within a system or between the system and external entities.

For information flow analysis, this paper adopts Sundresh's 'Information Engine' or 'Info-dynamic Engine' (Sundresh, 2000). A worked example of the use of the Infodynamic engine for a PSS has also been demonstrated (Durugbo et al., 2009). This Infodynamic engine is based on two interconnected or integrated subsystems using information already in their possession to generate a new piece of directly usable information.

Fig. 1: Information Engine Generation Cycle (Sundresh, 1997)

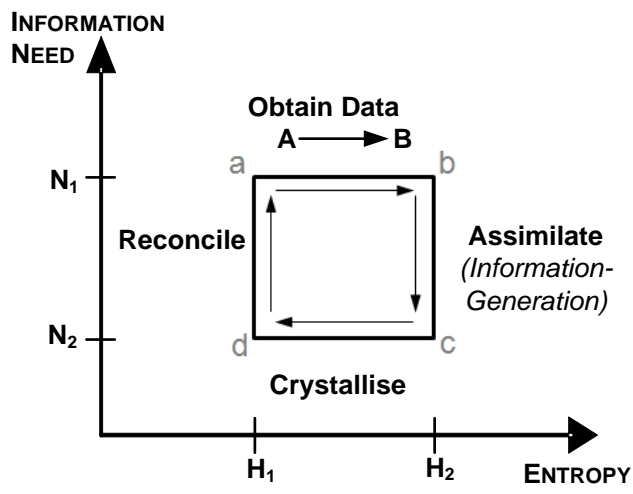

Four phases can be identified from the model shown in Fig. 1. In the first phase (a-b), $\mathrm{A}$ is designated the source and B is the recipient of the data. Next, B starts to process the data in isolation. The second stage $(b-c)$ relates to an information transformation stage in which information is processed with respect to the task to be done. At point $\mathrm{c}$ the data has now been transformed and begins the process of being separated, 'crystallized', into the parts usable and unusable for the specific application being considered. In the final step $(\mathrm{d}-\mathrm{a}), \mathrm{A}$ and $\mathrm{B}$ 'reconcile', i.e. realise a piece of information that B needs, based on the prior processes. These four phases of the Info-dynamic engine form the path for information flow.

Two measures are employed in the Info-dynamic engine: physical entropy and information need. The physical entropy can be computed as the sum of the algorithmic randomness and the statistical disorder of a given data $d$ i.e.

$$
S_{d}=K(d)+H_{d}
$$

Where $S_{d}$ is the physical entropy, $K(d)$ is the algorithmic randomness (or Kolmogorov complexity) of $d$ and $H_{d}$ is the statistical disorder that is dependent on the conditional probability of the data set.

Zudek (1989) refers to the statistical disorder in relation to missing information and the algorithmic randomness in terms of the known randomness (or disorder). The algorithmic randomness $(K(d))$ for a given data $d$ with length $d^{*}$ is defined as the shortest path that produces $d$ and is calculated as

$$
K(d) \equiv|d *|
$$


The statistical disorder $\left(H_{d}\right)$ provides information about microstates that are unavailable regardless of the presence of $d$. This statistical disorder can be computed as (Durugbo et al., 2009):

$$
H_{d}=-\sum_{k} P_{k \mid d} \log _{2} P_{k \mid d}
$$

Where $P_{k \mid d}$ is the conditional probability of the microstate $k$ given macrostate $d$

Each microstate $k$ can be described in terms of this conditional probability $\left(P_{k \mid d}\right)$ in relation to a macrostate $d$ which it constitutes. A microstate is described here as system configurations or arrangements that combine to give a system's macrostate. For instance, concentration of samples (analytes) and fluid dynamic phenomena (fluidics) can be thought of as microstates, for which fluids (gas and liquid) can be a macrostate.

'Information potential' is used for the model to describe information held by either A or $\mathrm{B}$, which the other needs to complete a task. An entity with a higher information potential can satisfy the needs of another entity with a lower information potential.

Information need is defined for the model as additional information (information over and above that already existing in the system) required for realising a goal or performing a task. Information need is equivalent to the probability of successfully carrying out a task without possession of information $m$ i.e. (Sundresh, 1997):

$$
N=P(\neg m)^{-1}
$$

Where $N$ is the information need, $\neg$ means 'without' or 'not' and $P$ is probability.

The reader is referred to Durugbo et al. (2009), Sundresh (1997) and Sundresh (2000) for the detailed arguments of information entropy and complexity on which this model is based.

\subsection{Link between data uncertainty assessment and information flow analysis}

While data uncertainty focuses on data collection, information flow considers information exchanges (Durugbo et al., 2009; Flanagan et al., 1989). Information flow is influenced by the transformation between data and information (Ackoff, 1989). Furthermore, the flow of information begins with the received order. Depending on the requirements, relevant information is captured and modelled for use in the project or organisation. The modelling helps to plan the information flow for a project or organisation.

This study identifies data uncertainty as the macrostate used to define information entropy in the Info-dynamic engine. To be able to analyse the efficiency of information flow, it is important to identify the types of uncertainties. This aims to establish the scope of uncertainties that influence data at a given time. The study takes the view that a suitable approach is necessary to recognise the ways to assess and analyse data uncertainties. As an outcome, increasing awareness will reduce the influence of data uncertainty.

The usual response to uncertainty challenges is to manage uncertainty using a framework (Erkoyuncu et al., 2009b). The five steps represented in Fig. 2 capture the main steps that are necessary from the identification of data uncertainty to its control over the duration of a given project. Each step has an overlap with the next and may require individuals to return to previous steps to make updates (e.g. in the light of new data) or to consider new uncertainties that had not previously been identified.

Data uncertainty management needs to capture the life cycle of a PSS in terms of the information requirement. This is because information requirements across the life cycle 
of a PSS may vary. Depending on the phase of the life cycle, the PSS could be based on either centralised or decentralised configurations. For instance, during the development phase the PSS may be based on a decentralised configuration to facilitate interactions between customer and manufacturer (Mont, 2002). Furthermore, for a new equipment scenario the manufacturer that developed the equipment may need to provide data related to the performance of the equipment. On the other hand, for an old equipment scenario, equipment usage and service data may also need to be collected. This necessitates information flow between the customer and the manufacturer. Subsequently, to be able to plan for a requirement it is important to collect (retrieve) data. Additionally, since not all data is reliable or useful (Flanagan et al., 1989), it is also necessary to process the data. This enables the structuring of available, useful and reliable data, which in essence means data gets transformed into information.

Fig. 2: Managing uncertainty (Erkoyuncu et al., 2009a)

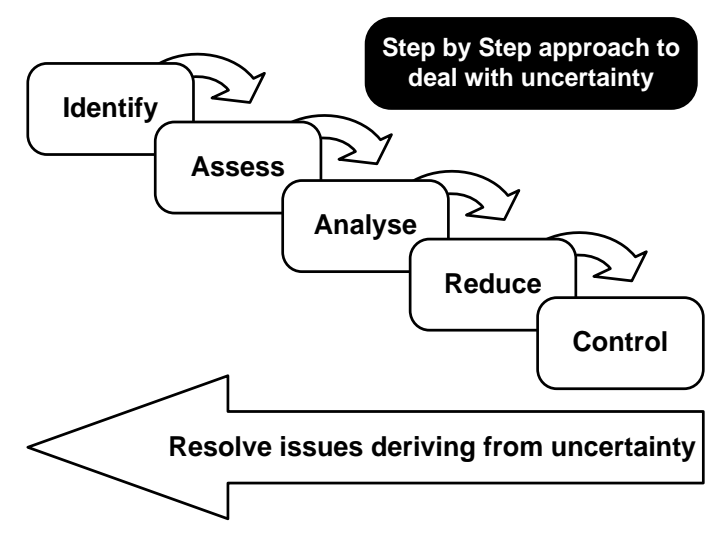

\section{Case Study: The Cranfield University Library System as a Product- Service System}

\subsection{Introduction to Case Study}

The Cranfield University Library (CUL) as a PSS contains integrated products (P1) and services (S1). The P1 offered by the CUL include books, reports, articles and magazines. S1 for the CUL include book borrowing, technical help to students, interlibrary loans, book purchasing, training, provision of specialist subject information, provision of offsite services.

The focus of the case study is on a function (interlibrary loan service) provided by the CUL. This approach to analysing decomposed parts of a system is consistent with function-orientation (Sommerville, 1992) which is an important approach to PSS design. The case study also focuses on the information system (that enables information flow) that enables the delivery of a PSS. This information system manages exchanges during interactions and negotiations between the CUL and students. The analysis of information flow within the interlibrary loan service function could aid the PSS implementer in 
planning and improving business operations in terms of supplier logistics, customer ability to correctly fill the interlibrary request form and so on.

The CUL was selected as a case study for familiarisation purposes, as it is an example that most researchers can relate to and it provides a suitable example to relate to within a PSS context. The CUL is based on a result-oriented approach (Tukker, 2004) through the delivery of interlibrary loan services. It is result oriented because it offers the opportunity to outsource (activity management) the delivery of journals to other libraries and sources, on behalf of the student. The interlibrary service to request a journal article has been selected for the case study for simplicity, because it concentrates the focus on a specific function of the library system.

\subsection{Data Uncertainty and Assessment}

In a library context, uncertainty is created while sharing data between the student making the request and the librarian who processes the data to deliver an order. Through interviews with the CUL it was identified that there are two major concerns in the process to fulfil an interlibrary loan request. First, the ability of the user to place an order for an interlibrary loan request. Second, for the library to request an interlibrary journal based on an order for an interlibrary loan request.

In the interviews, it was also highlighted that the first point as opposed to the second, created more data uncertainty issues in the provision of the interlibrary loan service. This reflects the significance of the input data on a request form. To further illustrate the steps taken to accomplish the first point, Table 2 represents the steps by which data is produced and its interaction with the library database. The user begins the process by identifying suitable journal papers, through the logon screen. The assessment based on keyword search leads to the thorough analysis of papers in order to realise whether a request will be handed into the library. Once a signature, to confirm the request is received the interlibrary loan request process begins with the library. At this point it was also mentioned that the used processes and tools (Kopak and M25 Consortium) to check availability of a given journal within the CUL and across other libraries provided reliable results, and did not create additional uncertainties. However, the only constraint included the case that journals across libraries needed to be catalogued in order for the search to provide reliable results, which was stated to be a small issue.

Table 2 Steps to place an interlibrary loan request

\begin{tabular}{lll}
\hline & User (Data) & System (Information Technology) \\
\hline Identify & User accesses database & Logon screen \\
Assess & Provide keyword & Search feature \\
Analysis & Description of material & Library catalogue \\
Mitigate & Request details & Order sheet \\
Control & Signature & Copyright sheet \\
\hline
\end{tabular}

Driven by the importance of the received data in fulfilling a loan request, the CUL provides training sessions to fill a request form. However, interpreting a request form appears to be the biggest challenge among all processes for the provision of this productservice system. The main data uncertainties associated with an interlibrary loan request 
include: rate of filling data into the wrong box (A1), rate of filling the wrong interlibrary request form (A2), rate of not filling enough data for the librarian to proceed with the search (A3), rate of not on system (A4), and rate of existence of interlibrary loan request in internal catalogue (A5).

\subsection{Information Flow and Analysis}

For the CUL as a PSS, with integrated processes for product P1 and services S1, an Info-dynamic engine can be demonstrated. The Info-dynamic engine is based on a predictive model of $\mathrm{P} 1$ i.e. the journal requested.

An information exchange during an interlibrary loan request for a journal offered by the CUL is presented in this paper. Six main steps are identified for a request by a student of an interlibrary loan for a journal article as shown in Fig. 3. First, the student searches the library catalogue for a journal. Second, the student places an order in the form for an interlibrary loan request. Third, the librarian logs the order. Fourth, the librarian searches internally and externally for the journal article. Fifth, the librarian confirms the order in relation to the journal information. Sixth, the librarian places an interlibrary loan request.

Fig. 3: Path in an information exchange for an interlibrary loan request (a.) Data flow diagram showing actual exchange (b.) Info-dynamic engine representation

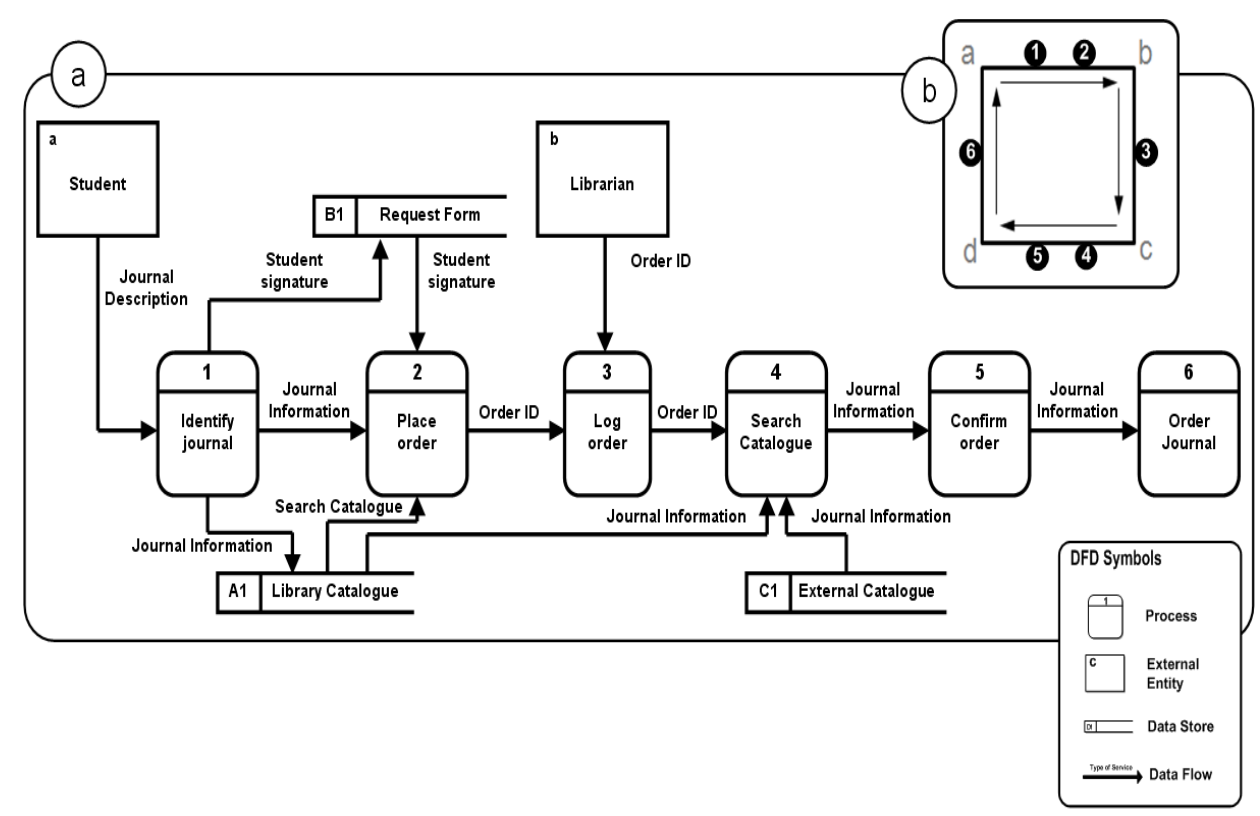

Three of the data uncertainties $(A S)$ identified in Section 4.2 are associated with requesting interlibrary loans - $\mathrm{A} 1, \mathrm{~A} 2$ and $\mathrm{A} 3$. Two other data uncertainties $(A O S)$ are also identified for confirming interlibrary loan requests - A4 and A5.

During the information exchange, seven pieces of information (UI) are required to uniquely identify an interlibrary request: user ID, journal title, article authors, year of 
publication, volume/part, ISSN (International Standard Serial Number) and page numbers.

Similarly, five additional pieces of information $(A I)$ are required for a request form: source of reference, declaration if article is for commercial use, copyright declaration, user comments and search type.

For an interlibrary loan journal to be provided, four pieces of information (IS) are required: article supplier, availability of requested article and location of article.

The macrostate in this case is data uncertainty while the microstates are $A S$ and $O A S$. Frequency of data uncertainty for the library system was automatically logged for two months and used to determine conditional probabilities (earlier identified in eqn. 3 ). Table 3 presents the conditional probabilities which have been logged for use in the case study.

Table 3 Conditional probabilities for Data uncertainty for interlibrary loan requests

\begin{tabular}{lcc}
\hline Process & Data Uncertainty & Conditional Probabilities \\
\hline Placing Interlibrary loans $(A S)$ & A1 & 0.06 \\
& A2 & 0.21 \\
Confirming Interlibrary loans (AOS) & A3 & 0.33 \\
& A4 & 0.12 \\
& A5 & 0.27 \\
\hline
\end{tabular}

The actual exchange is modelled by the data flow diagram shown in Fig. 3a. This case study examines the information flow for the library system. This can be mapped onto the Info-dynamic engine as shown in Fig. 3b. This is because the Info-dynamic engine is initiated by an 'obtain data' (a-b) phase as shown in Fig. 1 equates to the 'identify journal and 'place order' in Fig. 3a. The 'assimilation' (b-c) phase in which information is generated corresponds to the 'log order'. The crystallised data during phase c-d is used to accomplish 'search catalogue' and 'confirm order'. Similarly, phase $\mathrm{d}-\mathrm{a}$ in which $\mathrm{P} 1$ and S1 are reconciled corresponds to the 'order journal' step in Fig. 3a.

$H_{l}$ is the entropy at the point of the interlibrary request and $k$ (see eqn. 3) denotes the individual microstates for the journal (or macrostate) requested. $H_{2}$, in contrast, is the entropy at the point before the journal is ordered and $k$ denotes the individual microstates required to confirm the interlibrary request. As earlier mentioned the entropy is computed as the sum of the algorithmic randomness $\left(\mathrm{S}_{d}\right)$ and the statistical disorder $\left(\mathrm{H}_{d}\right)$ i.e. $\mathrm{S}_{d}+\mathrm{H}_{d}$

$N_{l}$ is information need at the point of the interlibrary request and the value of $m$ (see eqn. 4) is the number of information items required to distinguish a student user. $N_{2}$, on the other hand, is the information need at the point when a journal has been identified and is ready to be ordered. At this point, $m$ can be computed from both the information required to identify a particular student user and any other additional or internal information in the library system used to identify students.

For the journal to be requested, three uncertainties are possible i.e. $K(d)$ at $H_{l}$ is equal to 3. These uncertainties, A1, A2 and A3 have conditional probability values of 0.06 , 0.21 and 0.33 respectively (see Table 3 ). Consequently, from eqn. $3, H_{1}$ can be calculated as 


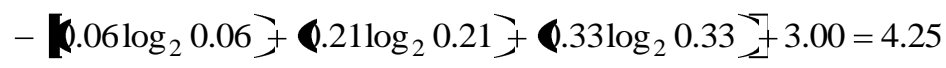

For the journal to be confirmed, all uncertainties associated with microstates $A S$ and $O A S$ are possible i.e. $K(d)$ at $H_{2}$ is equal to 5. Using Table 3 as before, the service request frequency values can be identified. From these parameters, $\mathrm{H}_{2}$ can be computed as

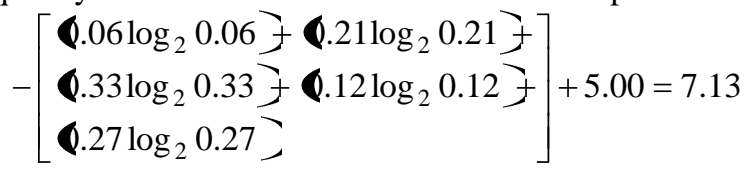

Total information in the exchange is the sum of information for journal provision (IS), request form completion $(A I)$ and information to uniquely identify a request (UI) i.e. $4+1+7=12$ (out of a possible 16). For this case, it is assumed that all information required to complete a task have the same probability. For $A I$, the main requirement is to complete a copyright declaration (student signature) for a journal by the product system P1. Additional information is then obtained to aid the delivery of the interlibrary loan service by S1. Consequently $m$ at $N_{l}$ equates to 0.75 . From this information, $N_{l}$ can be calculated as

$$
(1-0.75)^{-1}=4.00
$$

Similarly, the value of $N_{2}$ can be computed as $(1-0.25)^{-1}=1.33$

The value of $m$ at $N_{2}$ is 0.25 (or one quarter) because the only $I S$ information that is required from P1 by $\mathrm{S} 1$.

\section{Results- Data Uncertainty Assessment and Information Flow Analysis for Product-Service Systems}

\subsection{Scoring for assessing data uncertainty}

By implementing the Delphi technique with all three members of the CUL Interlibrary loan services the uncertainty assessment was performed. The NUSAP approach provided structure to the discussion as participants filled a pedigree matrix for the given set of uncertainties. The Delphi method aims to counteract the bias that results from the interaction between group members (Stewart et al., 1995). The approach elicits individuals' opinion and reason for a particular opinion separately, in order to reduce bias that could occur from other members in the group.

Table 4 Pedigree scores for case study

\begin{tabular}{ccccc}
\hline Uncertainty & Empirical & Methodological rigour & Validation & Pedigree score \\
\hline A1 & 0.42 & 0.25 & 0.25 & 0.31 \\
A2 & 0.50 & 0.67 & 0.50 & 0.56 \\
A3 & 0.25 & 0.42 & 0.25 & 0.31 \\
A4 & 0.75 & 0.75 & 0.67 & 0.72 \\
A5 & 0.75 & 0.92 & 0.75 & 0.81 \\
\hline
\end{tabular}

Along these lines the participants mutually agreed that $\mathrm{A} 1, \mathrm{~A} 2$ and $\mathrm{A} 3$, respectively, tend to be the most important uncertainties that have a larger influence on the interlibrary 
request process. Furthermore, the results of the pedigree scoring, as represented in Table 4 , highlighted that A1 and A3 constitutes the largest level of uncertainty and A2 accounts for the third largest uncertainty. The lower level uncertainties are A4 and A5 respectively. A pedigree score constitutes an average of the three criteria: empirical, methodological rigour and validation. Furthermore, values closer to 0 reflect higher uncertainties.

Given these results, the focus of uncertainty assessment is to identify those uncertainties that have high importance and hold a large level of uncertainty. A1, A2 and A3, as represented in Fig. 4, require additional effort to manage due to their importance and pedigree score.

Fig. 4: Main focus of uncertainty assessment

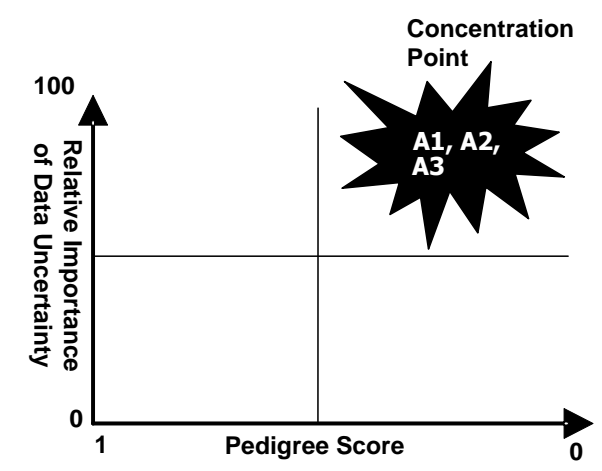

\subsection{Comparative Analysis for information flow}

As demonstrated, information exchange in the provision of the service for the interlibrary loan request can be mapped unto the Info-dynamic engine. However, the information exchange process could be further optimised based on the identified information flow path. For this reengineering process, two approaches have been suggested (Durugbo et al., 2009):

- Aggregating and restructuring information to reduce exchanges; or

- Reducing the number of actors and scenarios in the information flow system for which data collection and processes can be automated.

Both approaches as shown in Fig. 5 adopt separate schemes for improving information flow. The first approach considers the information exchanges during the interlibrary loan request while the second approach is based on the concentration point of the data uncertainty assessment of Fig. 4.

For the first approach (aggregating and restructuring information), the library information system can be configured as shown in Fig. 5a. Improved information flow can be achieved in a scheme that causes students to supply an ISSN number used to uniquely identify most journals. This ensures that a journal can be effectively identified by a student in the library (internal) catalogue.

In the second approach (reducing actors/scenarios), adequate automation facilities can be applied to eliminate four identified data uncertainties A1, A2 and A3. An integrated catalogue (internal and external) can be developed to reengineer information flow. This redesigned system (see Fig. 5b) can be configured for students to request journal 
information, search the integrated catalogue and place an interlibrary loan request. Data authentication can be applied to check that the inputs from users are correct. This approach eliminates data uncertainty A5 ensuring that a journal can be effectively identified and requested by a student in the library (internal) catalogue. A4 cannot be completely eliminated because it is dependent on the availability of the journal i.e. a journal ordered online may not be physically available at the external library where it was identified.

Fig. 5: Re-engineering the information flow (a.) aggregating and restructuring information (b.) reducing actors and scenarios (c.) optimised information flow

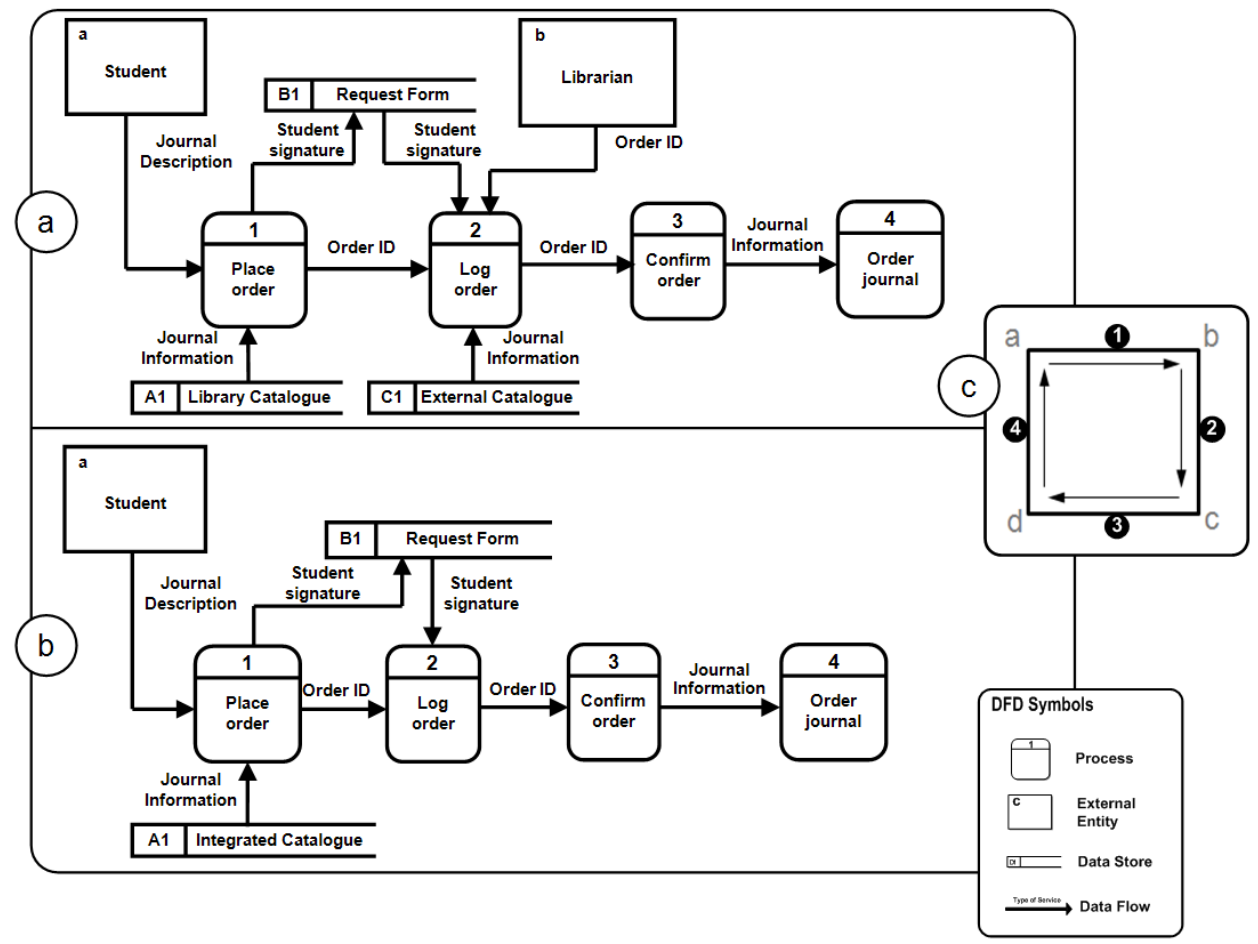

Information flow, in both approaches, as shown in Fig. 5c, is realised in four main steps. Step 1 involves placing an order for a journal. This request would also advise on the available orders and could allocate available resources as required. Step 2 involves logging the order to generate an order ID. Step 3 confirms the order to retrieve information about the requested journal. Step 4 places the order based on the identified source outside the CUL.

As part of the Info-dynamic engine, the value of the usable information can be calculated as the product of the information content and the information need i.e.

$$
\left(H_{2}-H_{1}\right) \cdot\left(N_{1}-N_{2}\right)
$$

Three Information flow efficiency concepts can also be derived based on the entropy change between $\mathrm{H} 1$ and $\mathrm{H} 2$ as well as the information need value difference between $\mathrm{N} 1$ and N2 (see Fig. 1). 
The first is the Information generation efficiency $(I G E)$ which compares the algorithmic information generated with the total information available i.e.

$$
I G E=1-H_{1} / H_{2}
$$

The second is the Information utilisation efficiency (IUE) which compares the algorithmic entropy for satisfied need with the initial need i.e.

$$
I U E=1-N_{2} / N_{1}
$$

The third Information flow efficiency concept is the Information system efficiency (ISE) which compares the information value generated with the total maximum information value in the data received. ISE compares actual use to possible use of information and is computed as:

$$
I S E=\left(H_{2}-H_{1}\right) \cdot\left(N_{1}-N_{2}\right) /\left(H_{2} \cdot N_{1}\right)
$$

In the current configuration for the CUL, the value of the usable information based on information need and physical entropy can be computed as (eqn. 5):

$(7.13-4.25) \cdot(4.00-1.33)=7.68$

The Information generation efficiency (IGE), Information utilisation efficiency (IUE) and Information system efficiency (ISE) can likewise be computed as (eqn. 6, 7, 8) 0.4, 0.67 and 0.27 respectively.

In the reengineered information flow, the efficiency measures applied are based on schemes to improve information exchanges (Fig. 5a) and to eliminate data uncertainty (Fig. 5b).

In the first approach (aggregating and restructuring information), the information flow focuses on the provision of an ISSN. This allows the librarian to concentrate on searching for articles on external catalogues. In terms of information need, $\mathrm{N}_{1}$ is now based on an ISSN and $I S$, while $\mathrm{N}_{2}$ is concerned with only $I S$. The value of the usable information remains constant and is calculated as:

$(7.13-4.25) \cdot(6.00-1.20)=13.82$

The new IGE, IUE and ISE can also be computed $0.40,0.80$ and 0.32 respectively.

In the second approach (reducing actors/scenarios), the system is configured to only accept valid requests with sufficient information. Since A1, A2 and A3 are eliminated, the value of $\mathrm{H}_{1}$ becomes 0 . The value of the usable information can then be computed as:

$$
(1.51-0) \cdot(4.00-1.33)=4.03
$$

The new IGE, IUE and ISE can also be computed as 1.00, 0.67 and 0.67 respectively.

\section{Discussion}

Within a PSS, the importance of customer value grows as the focus shifts away from the sale of an equipment towards it utilisation. Furthermore, existing research in PSS pays much attention to the process of defining suitable approaches to integrate products and services. However, it has been realised that there is a limited amount of research focusing on data and information flow. The significance of such research could be high, as the sustainability/continuation of customer value could depend on data and information flow, while influencing the system level of uncertainty. 


\subsection{Benefits of the Data Uncertainty Assessment and Information Flow Analysis}

One of the main benefits of this paper includes a simple measure to calculate the information flow efficiency (IGE, IUE and ISE) in a PSS, which was applied to a case study that focused on interlibrary loans at the CUL. Along with this to be able to realise suitable approaches to manage the rate of efficiency in information flow, this paper provides a method to assess data uncertainty. The rate of efficiency of the information flow depends on data uncertainty. The issue of data uncertainty and access to usable information is driven by the data collection process. Some of the commonly experienced issues include inconsistent data definitions in historical programs and the availability and accessibility of data. In the case study the Delphi approach was implemented to elicit the types of data uncertainty. The approach enabled the acquisition of a justifiable list of data uncertainties. Along with that, the NUSAP approach adopted in this paper offers the opportunity to understand those uncertainties that need further attention (e.g. better software to collect data, or promotion of particular data to be collected in a standard format). The three criterions selected, empirical, methodological rigour and validation offer a good base to capture the level of uncertainty for a given type of data uncertainty. Furthermore, the assessment of data uncertainty is expected to enhance awareness of issues and direct stakeholders to implement suitable strategies to eliminate or reduce data related issues. For instance based on the case study it became apparent that the importance of measures to reduce people filling in the wrong box on a interlibrary request form (A1), not filling enough data for the librarian to proceed with a request (A2) and the insufficiency of data on a request form (A3) appeared to be the three largest uncertainties that required attention. Furthermore, information flow analysis to eliminate these uncertainties will enable improvements in information flow efficiency.

Applying an information flow model such as the Info-dynamic engine in a PSS can be beneficial by offering an insight into system processes and for improving system efficiency. As demonstrated in the case study, a path for information flow can be established based on information exchanges in a PSS. This efficiency is driven by increasing value of generated usable information. Improved efficiency in the case study was realised based on identifying concentration points for data uncertainty and eliminating identified data uncertainties as shown in Table 5.

Table 5 Pedigree scores for case study

\begin{tabular}{lccc}
\hline Uncertainty & $\begin{array}{c}\text { Original } \\
\text { system }\end{array}$ & $\begin{array}{c}\text { Aggregation / } \\
\text { restructuring processes }\end{array}$ & $\begin{array}{c}\text { Reducing scenarios } \\
\text { and roles }\end{array}$ \\
\hline $\begin{array}{l}\text { Useable Information } \\
\text { Information Generation }\end{array}$ & 7.68 & 13.8 & 4.03 \\
$\begin{array}{l}\text { Efficiency (IGE) } \\
\text { Information Utilisation }\end{array}$ & 0.4 & 0.4 & 1 \\
$\begin{array}{l}\text { Efficiency (IUE) } \\
\text { Information System }\end{array}$ & 0.67 & 0.8 & 0.67 \\
Efficiency (ISE) & 0.27 & 0.32 & 0.67 \\
\hline
\end{tabular}

In the suggested 'information aggregation/restructuring' scheme, the efficiency of the information utilisation was optimised from an efficiency value (IUE) of 0.67 to 0.80 . Similarly, the efficiency of the information system was improved from an efficiency 
value (ISE) of 0.27 to 0.32 . Alternatively, optimisation of information flow could be realised in automation schemes to reduce actors and scenarios. This 'reduce actors and scenarios' approach further increased the overall efficiency of the information system (0.67) when compared to the original system (0.27) or the information aggregation and restructuring scheme (0.32).

These improvements are possible at the expense or tradeoffs for increased or decreased usable information. In the 'information aggregation/restructuring' scheme, usable information is increased to a value of 13.8 whereas in the 'reduce actors and scenarios' approach usable information is decreased to a value of 4.03 (see Table 5).

Additionally, the Pedigree scoring for data uncertainty helps to gain better understanding of information flow. The approach enhances the efficiency across processes within the architecture within the PSS (e.g. in delivering satisfactory interlibrary loan requests). Along with this, it offers the opportunity to conduct comparative analysis prior to a redesign scheme being put in place.

\subsection{Implications for the Design of Product-Service Systems}

Komoto et al. (2005) contend that the wide spread adoption of the PSS approach will ultimately depend on the establishment of technically sound modelling methodologies. Driven by this assertion, this paper has attempted to identify and apply data uncertainty and information flow for a PSS.

As shown in the case study, data uncertainty assessment and information flow analysis can be combined to improve specific functions of a PSS, and offer some useful measures for system performance. Some simple steps such as aggregating and restructuring processes could then be applied to eliminate uncertainty and improve efficiency of information flow.

In a PSS, the assessment of data uncertainty can influence the way in which the function-orientation can be carried out. It is the performance of each subsystem that feeds into the overall performance of satisfying customers through value delivery (Roy and Cheruvu, 2010). Data uncertainty represents the inability to rely on the assessment of the performance of a given subsystem. Furthermore, the level of data uncertainty highly depends on the data collection process. The stakeholders in the data collection process need to take into consideration issues such as accuracy and availability of data. The stakeholders within the data collection process also need to consider factors that influence the credibility of data. These may require consideration of data definitions, scope of historical data, and the sharing of data through corporate level software packages. This influences the organiser of a PSS to take measures that enable the delivery of value to the customer with higher certainty. Thus, the assessment of data uncertainty provides a suitable approach to consider the important subsystems that are prone to higher uncertainty. As a result, this will provide an approach to potentially redesign systems or develop new systems.

A PSS as an integrated solution must consider the interaction of units (products and services) from which it is composed. This process requires consideration for the interaction of system functions and the importance of each function in delivering the value in a PSS. Furthermore, this will need to capture the interactions across functions and understand the interdependencies between tasks in order to become aware of the combined influence of data uncertainty. Information flows could offer useful avenues for realising interactions in a PSS. Information exchanges in modern organisations are 
characterised by a collaboration of human judgement and computational facilities (Patel et al., 1996). Computation facilities are designed to cope with possibly large information quantities while human judgement is used in this context to consider the fulfilment of customer requirements without jeopardising business objectives of manufacturers. It is therefore important for PSS designers to consider how interacting units such as customers will make use of computation facilities to access, interpret and deliver information. For the case study of the CUL as a PSS, possible considerations could involve suggesting new journals to users based on profiles that are developed by the library.

\subsection{Limitation of the Study}

The Info-dynamic engine that has been used in the case study is a tool for modelling information flow at a high-level of abstraction. Furthermore, the tool is limited to a sequential representation of information flow extracted from information exchanges (Durugbo et al., 2009). The engine analyses information flow for the delivery of a PSS but factors such as criticality or timing of the information for the delivery of a service are neither identified nor supported. It is also vital to note that a comprehensive approach to modelling information is also required to define aspects of information such as information structure and information content.

Adopting the NUSAP approach necessitates some form of training for the participants to score the criteria within the Pedigree matrix. The case study showed that participants at the library found difficulties scoring the criterion within the limited time that was allocated. Furthermore, the approach due to the limited amount of available data, uncertainty requires human judgment to identify the causes and types of data uncertainty and inefficiencies in information flow.

\section{Conclusions}

This paper proposes an approach for assessing data uncertainty and for analysing information flow in a PSS, for which a library system was selected as a case study. An integrated approach was adopted based on data uncertainty during data collection and information flow during information exchanges. Furthermore, it is envisaged that by adopting the methodology suggested in this paper it will be possible to improve the delivery of a PSS. This was demonstrated in the paper by means of a prioritisation of data uncertainty and enhancement of the efficiency of information flow for the PSS library case study.

The case study involved three main steps for a library system that focused on interlibrary loans. Firstly the Delphi method was applied to capture data uncertainties that arise in delivering the interlibrary loan service. This step also involved the development of data flow diagrams to represent information exchanges. Secondly, the paper implemented the NUSAP approach to rank uncertainties. For this purpose, the types of uncertainties that were identified through the Delphi approach were focused on. Thirdly, the Info-dynamic Engine was applied to compare the efficiency of the original system and to reengineer the system based on the uncertainties that were identified. The case study suggests that a combination of data uncertainty and information flow analysis could be applied to improve a specific function of a PSS. 
For future work, the research will look into improvements for the methods that have been suggested in this paper. Along with this, further considerations are necessary to consider the gap and synergy between information flow and data uncertainty. The case study fits into the use-oriented context of a PSS, it would also be useful to consider the approach for product and result oriented models in a PSS. In terms of the case study the interest could expand from the interlibrary loan focus to capture other products and services that are offered within the library.

\section{References}

Ackoff, R. (1989) 'From Data to Wisdom', Journal of Applied Systems Analysis, Vol. 16, pp. 3-9.

Baines, T., Lightfoot, H. W., Evans, S., Neely, A., Greenough, R., Peppard, J., Roy, R., Shehab, E., Braganza, A., Tiwari, A., Alcock, J. R., Angus, J. P., Bastl, M., Cousens, A., Irving, P., Johnson, M., Kingston, J., Lockett, H., Martinez, V., Michele, P., Tranfield, D., Walton, I. M., and Wilson H. (2007) 'State-of-the-art in product-service systems', Proceedings of the Institution of Mechanical Engineers, Part B: Journal of Engineering Manufacture, Vol. 221, No. 10, pp. 15431552.

Boussabaine, A. and Kirkham R. (2004) 'Whole Life-cycle costing: Risk and risk responses (1st Edition)', Oxford: Blackwell Publishing, pp. 56-81

Buganza, T. and Verganti, R. (2006) 'Life-Cycle Flexibility: How to measure and improve the innovative capability in turbulent environments', Journal of Product Innovation Management, Vol. 23, pp. 393-407.

Coulon, R., Camobreco, V., Teulon, H. and Besnainou, J. (1997) 'Data quallity and uncertainty in LCI', International Journal of Life Cycle Assessment, Vol. 2, No. 3, pp.178-182

Curran, R., Raghunathan, S. and Price M. (2004) 'Review of aerospace engineering cost modelling: The genetic causal approach', Progress in Aerospace Sciences, Vol. 40, No. 8, pp. 487-534.

DeMarco, T. (1979) 'Structured Analysis and System Specification', New Jersey: Yourdon Press.

Durugbo, C., Tiwari, A. and Alcock, J. R. (2009) 'An Infodynamic Engine Approach to Improving the Efficiency of Information Flow in a Product-Service System', Proceedings of the 1st CIRP IPS2 Conference, pp. 107-112.

Durugbo C., Tiwari A., Alcock J.R., (2010a), 'Managing Information Flows for Product-Service Systems Delivery', Proceedings of the 2nd CIRP IPS2 Conference, 365-370

Durugbo, C., Tiwari, A. and Alcock, J. R. (2010b) 'A Review of Information Flow Diagrammatic Models for Product-Service Systems', International Journal of Advanced Manufacturing Technology (Accepted for publication).

Erkoyuncu, J. A., Roy, R., Shehab, E. and Cheruvu, K. (2010) 'Understanding Service Uncertainties in Industrial Product- Service System Cost Estimation', International Journal of Advanced Manufacturing Technology (Accepted for publication).

Erkoyuncu, J. A., Roy, R., Shehab, E. and Wardle, P. (2009) 'Uncertainty challenges in service cost estimation for product- service systems in the aerospace and defence industries', Proceedings of the 1st CIRP IPS2 Conference, pp. 200-207.

Flanagan, R., Norman, G., Meadows, J. and Robinson, G. (1989) 'Life cycle costing-Theory and practice', London: Blackwell Scientific Publication-BSP Professional Books.

Funtowicz, S.O., Ravetz J.R. (1990) 'Uncertainty and Quality in Science for Policy', Dordrecht: Kluwer.

Komoto, H., Tomiyama, T. and Nagel, M. (2005) 'Life Cycle Simulation for Analyzing Product Service Systems', Fourth International Symposium on Environmentally Conscious Design and Inverse Manufacturing, pp. 386-393. 
Meade, P. T. and Rabelo, L. (2004) 'The technology adoption life cycle attractor: Understanding the dynamics of high-tech markets', Technological Forecasting and Social Change, Vol. 71, No. 7, pp. 667-684.

Mont, O. K. (2002) 'Clarifying the concept of product-service system', Journal of Cleaner Production, Vol. 10, No. 3, pp. 237-245.

Morelli, N. (2006) 'Developing new product service systems (PSS): methodologies and operational tools', Journal of Cleaner Production, Vol. 14, No. 17, pp. 1495-1501.

Ormerod, T. C. and Shepherd, A. (2004) 'Using task analysis for information requirements specification: The SGT method', In: Diaper, D. and Stanton, N. (ed.), The Handbook of Task Analysis for Human-Computer Interaction, (pp. 347-366), London: Lawrence Erlbaum Associates.

Patel A., Meade R., O'Sullivan D., Tierney M., (1996) 'Information modelling for service provisioning - the DESSERT experience', Computer Standards and Interfaces, Vol. 18, No. 2, pp. 175-189.

Refsgaard, J.C., Sluijs, J.P., Hojberg, A.L., Vanrolleghem, P.A. (2007) 'Uncertainty in the environmental modeling process-A framework and guidance', Environmental Modelling and Software, Vol. 22, No. 11, pp. 1543-1556.

Roy, R. and Cheruvu, K. (2010) 'A competitive framework for Industrial Product Service Systems', International Journal of Internet Manufacturing and Services, Vol. 2, No. 1, pp. 4-29.

Scheller, A. (1990) 'Information modelling for distributed applications', IEEE Workshop on Future Trends of Distributed Computing Systems, pp. 494-500.

Sommerville, I. (1992) Software Engineering, 4th edn. Addison Wesley, London

Stewart, R.D., Wyskida, R.M., Johannes, J.D. (1995) 'Cost estimator's reference manual', New York: John Wiley, pp. 267-285.

Sundresh, T. S. (1997) 'Information complexity, information matching and system integration', Proceedings of the IEEE International Conference on Systems, Man and Cybernetics, Vol. 2, pp. $1826-1831$.

Sundresh, T. S. (2000) 'Information flow and processing in anticipatory systems', IEEE International Conference on Systems, Man and Cybernetics, Vol. 1, pp. 271-276.

Tukker, A. (2004) 'Eight types of product-service system: eight ways to sustainability? Experiences from SusProNet', Business Strategy and the Environment, Vol. 13, No. 4, pp. 246-260.

Van der Sluijs, J.P., Risbey, J.S., Kloprogge, P., Ravetz, J.R., Funtowicz, S.O., Quintana, S.C., Pereira, A.G., De Marchi, B., Petersen, A.C., Janssen, P.H.M., Hoppe, R., Huijs, S.W.F., (2003) 'RIVM/MNP Guidance for Uncertainty Assessment and Communication: Detailed Guidance. Copernicus Institute for Sustainable Development', Utrecht University, Utrecht, the Netherlands; online at: http://www.nusap.net/downloads/detailedguidance.pdf.

Van der Sluijs, J.P., Craye, M., Funtowicz, S.O., Kloprogge, P., Ravetz, J., Risbey, J. (2005) 'Combining quantitative and qualitative measures of uncertainty in model based foresight studies the NUSAP system', Risk Analysis, Vol. 25, No. 2, pp. 481-492.

Von Krauss, M.P.K., Kaiser, M., Almaas, V., van der Sluijs J.P., Kloprogge, P. D., (2008) 'Diagnosing and prioritizing uncertainties according to their relevance for policy: The case of transgene silencing', Science of the Total Environment, Vol. 390, No. 1, pp. $23-34$.

Westkämper, E., Alting, L. and Arndt, G. (2000) 'Life Cycle Management and Assessment: Approaches and Visions towards Sustainable Manufacturing (keynote paper)', CIRP Annals Manufacturing Technology, Vol. 49, No. 2, pp. 501-526.

Zurek, W. H. (1989) 'Algorithmic randomness and physical entropy', Physical Review, Vol. 40, No. 8, pp. 4731-4751 